\title{
Influencia de los trastornos mentales y de la personalidad en el abandono de procesos en personas sin hogar
}

Carlos Salavera

Universidad de Zaragoza (España)

\begin{abstract}
Resumen: Las personas sin hogar protagonizan un alto índice de abandonos en los procesos terapéuticos que establecen. El motivo de abandono no siempre es conocido, en esta investigación se aborda si viene mediado por los trastornos mentales o son los trastornos de personalidad los que llevan un mayor peso. Los resultados muestran a las personas sin hogar con deterioro cognitivo leve y trastornos de personalidad del grupo de problemas interpersonales y ambivalentes como los principales candidatos al abandono de procesos de corte psicosocial. Esto implica la necesidad de tener en cuenta estos trastornos en el tratamiento y desarrollo de programas específicos de intervención.

Palabras clave: Ejes; tratamiento; abandono; persona sin hogar.
\end{abstract}

\section{Introducción}

Las personas sin hogar viven en las calles de las ciudades, temporalmente en albergues, a causa de una ruptura encadenada, brusca y traumática de sus lazos familiares, sociales y laborales (Cabrera, Malgesini y López, 2003). Son uno de los grupos más vulnerables y desfavorecidos de la sociedad (Henry, Boyer, Belzeaux, Baumstarck-Barrau y Samuelian, 2010; Pascual et al., 2008; Speak, 2013). Además, en los últimos años (2005-2012) la población sin hogar se ha incrementado en España un $132 \%$, falleciendo en nuestro país una persona sin hogar cada cinco días (INE, 2012). Se asocian a una baja calidad de vida y altas tasas de enfermedad física y psíquica (Folsom et al., 2005; Sullivan, Burman, Koegel y Hollenberg, 2000), sufren un evidente deterioro mental fruto de su estancia en la calle (Csémy, Vágnerová y Marek, 2011; Muñoz, Vázquez, Panadero y Vázquez, 2003; Salavera, Puyuelo y Orejudo, 2009; Solliday-McRoy, Campbell, Melchert, Young y Cisler, 2004) y se consideran el nivel máximo de exclusión social que se produce en una sociedad moderna (Combaluzier y Pedinielli, 2003; Vágnerová, Csémy y Marek, 2012).

La adherencia a tratamientos psicosociales en esta población es muy baja, convirtiéndose en muchas ocasiones en actuaciones puntuales sobre las necesidades más básicas (comida, alojamiento y ropa). Cuando consiguen iniciar procesos psicosociales rehabilitadores el abandono de tratamiento es elevado, impidiendo la consecución de los objetivos terapéuticos (Twenge, Baumeister, DeWall, Ciarocco y Bartels, 2007).

En los últimos años se han realizado numerosos estudios sobre abandono de tratamiento en poblaciones clínicas o en tratamiento de adicciones (Combaluzier, Gouvernet y

* Dirección para correspondencia [Correspondence address]: Carlos Salavera. Departamento de Psicología y Sociología. Universidad de Zaragoza. c/San Juan Bosco, 7. 50009 Zaragoza (España). E-mail: salavera@unizar.es
Title: Influence of mental disorders and personality problems processes in the abandonment of homeless people.

Abstract: Homeless people have a high rate of dropouts in establishing therapeutic processes. The reason for abandonment is not always known, this research addresses whether is mediated by mental disorders or personality disorders are those that carry more weight. The results show homeless people with mild cognitive impairment and personality disorders group interpersonal problems and ambivalent as the leading candidates to abandon psychosocial cutting processes. This implies the need to take into account in treating these disorders and development of specific programs of intervention.

Key words: Axles; treatment; abandonment; homeless person.

Bernoussi, 2009; Villeneuve, Potvin, Lesage y Nicole, 2010), no tanto en personas sin hogar. En esta población, se achacan estos abandonos a los trastornos de personalidad (TP), una de las patologías mentales presentes en esta población, sin observar los otros ejes clínicos que pueden explicar más ampliamente los motivos de abandono de las personas sin hogar. Desde el punto de vista del modelo multiaxial, el Eje I (sindromes clínicos, compuesto por los trastornos mentales), puede completar la explicación que desde el Eje II (trastornos de personalidad, características desadaptativas de la personalidad) se ha venido dando sobre las causas de abandono de tratamientos de corte psicosocial en las personas sin hogar (Salavera, Puyuelo, Tricás y Lucha, 2010; Samuel, Connoly y Ball, 2012). En este estudio se analizó qué factores (síndromes clínicos o trastornos de personalidad) tienen más peso en el abandono de tratamientos psicosociales en las personas sin hogar.

\section{Método}

\section{Participantes}

La muestra está compuesta por personas sin hogar $(N=$ 89) que realizaron en el centro un proceso de inserción, con tratamiento psicosocial y clínico. Los 89 sujetos del estudio, fueron seleccionados entre 93 personas, descartándose dos casos por desestabilización psicopatológica y otros dos por abandono mientras se efectuaba el estudio. Todos los participantes, pasaron por un proceso de inserción para personas sin hogar, en función de los siguientes criterios: a) cumplir el criterio de persona sin hogar; b) llevar más de dos meses en el centro; c) participación voluntaria en el estudio; y d) permanecer el tiempo necesario para completar el mismo.

\section{Medidas de evaluación}

Entrevista de Valoración Inicial. Al principio se realizó una entrevista individual estructurada para realizar el diagnóstico. 
En esta entrevista se recogían los datos más significativos: edad, estado civil, nivel de estudios, edad de inicio del transeuntismo, motivo del mismo, relaciones familiares, consumos de alcohol y sustancias y tratamiento psicológico anterior.

CIDI. Composite International Diagnostic Interview (WHO, 1997). Se trata de una entrevista estructurada que utiliza criterios DSM. La CIDI incluye en su totalidad el DIS (Diagnostic Interview Schedule) y el MSE (Minimental State Exam). Como es habitual en los estudios con personas sin hogar (PSH), no se utilizó el CIDI en su totalidad. Se utilizaron únicamente los módulos: Trastorno depresivo mayor (de episodio único o de episodios recurrentes), Trastorno distímico, Deterioro cognitivo, Trastorno esquizofrénico, Trastorno esquizofreniforme y Trastornos por uso de alcohol o sustancias psicoactivas.

Inventario Clínico Multiaxial de Millon (MCMI II) (Millon, 1997). El cuestionario consta de 175 preguntas, con estructura verdadero-falso, que se contesta en 25-30 minutos. El resultado nos da 10 escalas básicas de personalidad: esquizoide, fóbica o evitativa, dependiente, histriónica, narcisista, antisocial, agresivo-sádica, compulsiva, pasivo-agresiva y autodestructiva; 3 escalas de personalidad patológica: esquizotípica, límite y paranoide; seis síndromes clínicos de gravedad moderada: ansiedad, histeriforme, hipomanía, neurosis depresiva, abuso de alcohol y abuso de drogas; y tres síndromes clínicos de gravedad elevada: pensamiento psicótico, depresión mayor y trastorno delirante.

Entrevistas de Valoración de los trastornos Eje I y II del DSM$I V$-TR. Se realizaron una serie de entrevistas individuales estructuradas para el diagnóstico de la problemática y los trastornos de los ejes I y II.

\section{Procedimiento}

El estudio se realizó por medio de entrevistas de dos psicólogos clínicos, que realizaron la entrevista de Valoración Inicial. Para medir los trastornos del eje II del DSM-IV-TR, tras analizar distintos instrumentos de evaluación de la personalidad, se seleccionó el MCMI II: Inventario Multiaxial Clínico de Millon II (Millon, 1997), por su facilidad y rapidez de aplicación, así como la posibilidad de obtener gran cantidad de información relevante sobre los sujetos y por la existencia de estudios previos con esta población, que permitiesen su comparación. Por último, para la valoración y diagnóstico de la problemática existente en el eje I y II se realizaron una entrevistas diagnósticas, en la que se analizaron los problemas presentes en dichos ejes.

Todos los sujetos eran varones, mayores de 18 años y con un recorrido en el proceso lo suficientemente amplio como para poder recoger los datos y hacer una valoración objetiva de los mismos. Además firmaron un consentimiento informado de participación en el estudio y se respetaron las normas éticas siguiendo la Declaración de Helsinki.

En el estudio se ha considerado la presencia de un trastorno de personalidad cuando la puntuación en la tasa-base
(TB) del MCMI II es superior a 74. Antes de comenzar el estudio, con cada participante, se esperó un tiempo superior a dos meses de estancia en el centro, tiempo considerado esencial, para medir adherencia al tratamiento y conseguir sinceridad y fiabilidad en las entrevistas y pruebas administradas.

Para el análisis estadístico de los datos, se utilizó el programa estadístico SPSS, en su versión 15.0. Se realizó un análisis descriptivo (máximos, mínimos, medias y desviación estándar) para cada una de las variables. En todos los casos se trabajó con un nivel de significación del 5\%. Se realizaron análisis de variables cruzadas y correlaciones a nivel bilateral.

\section{Resultados}

\section{Variables sociodemográficas}

En este apartado (Tabla 1), destaca el alto número de sujetos jóvenes sin hogar en un proceso de inserción, casi una de cada cuatro personas es menor de 30 años, lo que indica un pronto comienzo en el fenómeno del transeuntismo; por otro lado, sorprende el bajo porcentaje de mayores de 50 años, tan sólo un $7.9 \%$, que puede indicar el poco interés de personas de esta edad en procesos de inserción, sin duda por el deterioro sufrido en su estancia en la calle y el desencanto ante procesos anteriores. Respecto a su estado civil, aunque el $60.7 \%(n=47)$ son solteros, esto no quiere decir que no hayan tenido pareja previa, incluso en alguna ocasión son padres de hijos no reconocidos o sin carga de manutención, aunque destaca el bajo nivel de personas con pareja durante su estancia en el centro, tan sólo un 3.3\% y alto nivel de ruptura en las personas que han tenido pareja, un $36 \%$. Pero por encima de todos los datos, sobresale el número de personas que no han establecido una relación estable con su pareja. Estos datos son coherentes con otros estudios (Längle, Egerter, Albretcht, Petrasch y Buchkremer, 2005; Muñoz et al., 2003; Vágnerova et al., 2012). En cuánto a estudios realizados vemos el bajo nivel de escolarización, principalmente por comienzo de su incorporación al mercado laboral.

\begin{tabular}{|c|c|c|c|}
\hline & & $N$ & $\%$ \\
\hline \multirow[t]{6}{*}{$\overline{\text { EDAD }}$} & Media & 37.86 & \\
\hline & Rango & $(22-52)$ & \\
\hline & $<30$ años & 19 & $(21.3 \%)$ \\
\hline & 30-39 años & 32 & $(36 \%)$ \\
\hline & 40-49 años & 31 & $(34.8 \%)$ \\
\hline & $>50$ años & 7 & $(7.9 \%)$ \\
\hline \multirow[t]{3}{*}{ ESTADO CIVIL } & Solteros & 54 & $(60.7 \%)$ \\
\hline & Separados/divorciados & 32 & $(36 \%)$ \\
\hline & Parejas de hecho & 3 & $(3.3 \%)$ \\
\hline \multirow[t]{4}{*}{ ESCOLARIDAD } & Certificado escolaridad & 31 & $(35.2 \%)$ \\
\hline & EGB & 42 & $(47.2 \%)$ \\
\hline & FP & 10 & $(11.2 \%)$ \\
\hline & BUP & 5 & $(5.6 \%)$ \\
\hline
\end{tabular}




\section{Variables del eje I}

En este apartado se encontró que 15 de las PSH participantes en el estudio padecían Depresión mayor, lo que supone un $20.22 \%$ de la muestra. En concreto, 13 personas obtuvieron un diagnóstico de depresión mayor de episodio único, 5 de depresión recurrente, 6 distimia (6.74\%) y 15 trastornos afectivos $(16.85 \%)$. En cuanto a trastornos psicóticos, 7 personas $(7.86 \%)$ según esta entrevista tenían un diagnóstico de Esquizofrenia y no se encontró a nadie con trastorno esquizofreniforme. En lo referente al deterioro cognitivo, presentaban episodios amnésicos 3 de los participantes $(3.37 \%)$, en grado moderado y ninguno en su forma grave. Por último, 46 presentaban Dependencia o abuso de alcohol $(51.68 \%)$ y 33 una dependencia o abuso de sustancias psicoactivas $(37.07 \%)$.

Todos los diagnósticos fueron confirmados por medio de entrevistas individualizadas efectuadas por del psicólogo clínico del centro. Los participantes, en todos los casos, mostraban estabilidad psicopatológica en el momento de su participación en el estudio, descartándose dos casos por descompensación.

\section{Variables eje II}

En cuanto a los resultados obtenidos (tabla 2) del Inventario Clínico Multiaxial de Millon (MCMI II) (Millon, 1997), se observa como los trastornos antisocial $(38.2 \%, n=34)$, compulsivo $(32.6 \%, n=29)$, dependiente $(28.1 \%, n=25)$, esquizoide y narcisista $(27 \%, n=24)$, son los que obtuvieron puntuaciones más altas, considerando TB $>74$. Hay que señalar que hubo sujetos que en su prueba se reflejó que pueden tener una o más subescalas con puntuaciones altas.

Tabla 2. Puntuaciones MCMI II $(N=89)$.

\begin{tabular}{lccccc}
\hline & Mínimo & Máximo & Media & D.S. & $\%$ TB $>74$ \\
\hline Esquizoide & 0 & 117 & 59.98 & 26.543 & $24(27 \%)$ \\
Fóbico & 2 & 103 & 50.82 & 29.466 & $22(24.7 \%)$ \\
Dependiente & 0 & 108 & 55.93 & 29.523 & $25(28.1 \%)$ \\
Histriónico & 5 & 100 & 51.66 & 24.518 & $18(20.2 \%)$ \\
Narcisista & 0 & 109 & 56.61 & 24.982 & $24(27 \%)$ \\
Antisocial & 0 & 121 & 65.84 & 28.324 & $34(38.2 \%)$ \\
Agresivo & 0 & 120 & 54.64 & 28.161 & $19(21.3 \%)$ \\
Compulsivo & 5 & 120 & 65.82 & 26.168 & $29(32.6 \%)$ \\
Pasivo & 0 & 103 & 41.93 & 27.971 & $13(14.6 \%)$ \\
Autodestructivo & 0 & 109 & 52.28 & 25.259 & $12(13.5 \%)$ \\
Esquizoide & 5 & 117 & 55.70 & 25.988 & $18(20.2 \%)$ \\
Límite & 0 & 112 & 45.48 & 27.667 & $11(12.4 \%)$ \\
Paranoide & 8 & 118 & 62.43 & 22.869 & $19(21.3 \%)$ \\
\hline
\end{tabular}

También se analizó el número de trastornos de personalidad de cada sujeto, se aprecia que había sujetos en tratamiento de inserción para personas sin hogar sin ningún trastorno de personalidad (19.5\% de los casos, $n=15)$, con un trastorno de personalidad $(23.4 \%, n=18)$, con dos trastornos $(18.2 \% ; n=14)$ y con tres o más trastornos de personalidad presentes $(39 \% ; n=30)$.

Además se analizó el peso de los ejes I y II tuvieron sobre el grado de abandono de los procesos. En los participan- tes en el estudio, 33 personas abandonaron (37.08\%), mientras completaron el proceso un total de $56(62.92 \%)$. Por último, se analizó si el abandono venía determinado por padecer unos trastornos u otros (tabla 3) en la que se muestran los trastornos mostrados por los sujetos, así como los porcentajes que suponen sobre abandonos e inserciones.

\begin{tabular}{|c|c|c|c|}
\hline & $\begin{array}{c}\text { Trastornos } \\
\text { mostrados sobre } \\
\text { los abandonos }\end{array}$ & F & $p$ \\
\hline Depresión recurrente & $4(12.12 \%)$ & .255 & .615 \\
\hline Depresión episodio único & $1(3.03 \%)$ & .652 & .421 \\
\hline Distimia & $2(6.06 \%)$ & 1.154 & .286 \\
\hline J Trastorno afectivo & $3(9.09 \%)$ & 237 & .627 \\
\hline II Esquizofrenia & $9(10.11 \%)$ & 2.273 & .135 \\
\hline Deterioro cognitivo & $3(9.09 \%)$ & 5.474 & .022 \\
\hline Dependencia de alcohol & $21(63.63 \%)$ & 3.035 & .085 \\
\hline Dependencia de sustancias & $13(39.39 \%)$ & .118 & .732 \\
\hline$\overline{\text { Esquizoide }}$ & $12(36.36 \%)$ & 3.925 & .051 \\
\hline Fóbico & $10(30.30 \%)$ & 1.772 & .187 \\
\hline Dependiente & $4(12.12 \%)$ & 6.670 & .011 \\
\hline Histriónico & $8(24.24 \%)$ & 1.039 & .311 \\
\hline Narcisista & $11(33.33 \%)$ & .608 & .438 \\
\hline$\exists$ Antisocial & $16(48.48 \%)$ & 2.893 & .093 \\
\hline$\approx$ Agresivo & $7(21.21 \%)$ & .828 & .365 \\
\hline [I] Compulsivo & $10(30.30 \%)$ & 1.268 & .263 \\
\hline Pasivo & $7(21.21 \%)$ & 1.632 & .205 \\
\hline Autodestructivo & $6(18.18 \%)$ & .090 & .765 \\
\hline Esquizotípico & $9(10.11 \%)$ & 2.112 & .150 \\
\hline Límite & $7(21.21 \%)$ & 3.874 & .046 \\
\hline Paranoide & $10(30.30 \%)$ & .061 & .805 \\
\hline
\end{tabular}

En el caso de los trastornos mentales, tan sólo en el deterioro cognitivo se encontró significación $\left(F=5.474^{*}\right)$. En los trastornos de personalidad sólo las personas con trastorno dependiente $\left(F=6.992^{*}\right)$ y límite $\left(F=3.874^{*}\right)$ mostraban relación con el abandono del proceso, si bien lo hacían en distinto sentido, mientras la persona con TDP mostraba mayor adherencia al proceso y menor índice de abandonos, en los sujetos con TLP se reflejaba como motivo de abandono.

\section{Discusión y conclusiones}

El estudio se centró en buscar la causa de abandono de proceso, intentando valorar si eran los trastornos mentales o de la personalidad presentes en las personas sin hogar, los que determinaban éste.

En cuanto al Eje I, con los datos obtenidos en la investigación, se encontró que en los abandonos, aun cuando las frecuencias eran mayores en consumos de alcohol y otras sustancias, el factor más determinante en el abandono era el deterioro cognitivo de la persona, en la que a pesar de su baja presencia se muestra relevante en la determinación de abandono de los procesos terapéuticos, cuestión concordante con estudios anteriores (Herrman, 2008; Muñoz, Vázquez, 
Bermejo, Vázquez y Sanz, 1996; Notaro, Khan, Kim, Nasaruddin y Desai, 2013; Solliday-McRoy et al., 2004).

Respecto al eje II, los sujetos participantes padecen preferentemente los trastornos pertenecientes al grupo de problemas interpersonales y ambivalentes en conflicto. Los trastornos más presentes resultaron ser: antisocial, compulsivo, dependiente, narcisista y esquizoide, con porcentajes similares a los encontrados en anteriores estudios (Fazel, Khosla, Doll y Geddes, 2008; Längle et al., 2005; Salavera, 2009). Como era de esperar, los trastornos más presentes en la muestra estaban relacionados con las personalidades con problemas interpersonales (Dependiente, Narcisista y Antisocial), junto con uno perteneciente a las personalidades ambivalentes con conflictos (Compulsivo). Esto analizado desde la tipología de Millon (1998), refleja una persona que busca la independencia no desde la propia autoconfianza, sino desde la desconfianza en los demás, con frecuentes fracasos en sus obligaciones y de comportamiento irresponsable y trasgresor (TAP), expresivamente arrogante e interpersonalmente explotador, sintiéndose por encima de las normas de convivencia (TNP), con graves escisiones internas de las que no pueden escapar (TCP), evitando las responsabilidades adultas y la autoafirmación, careciendo de competencias funcionales (TDP). En cuanto al eje II, como principal conclusión, se observa en el estudio una alta prevalencia de TP, muy por encima de los datos epidemiológicos encontrados en población general (DSM-IV-TR, 2000; INE, 2011) y que influiría en el abandono del proceso.

Los resultados de este estudio, muestran que las personas sin hogar examinadas, presentan una mayor sintomatología psicopatológica en el eje II que la encontrada en población general, en línea con estudios anteriores (Berghuel, Levesque y Pakzad, 2013; Combaluzier et al., 2009; Samuel et al., 2012), determinando el abandono de los procesos. Sin embargo, esta cuestión no tan está presente en el eje I, aún cuando investigaciones anteriores parecen ir en el sentido de

\section{Referencias}

American Psychiatric Association, (2004). Manual diagnóstico y estadístico de los trastornos mentales (4 ${ }^{a}$ ed. Revisada). Barcelona: Ed. Masson.

Bergheul, S., Levesque, S. \& Pakzad, S. (2013). La criminalisation des itinérants au Canada, La Revue de Médecine Légale, 4 (1), 45-51

Cabrera, P. J., Malgesini, G. y López, J. A. (2003). Un techo y un futuro: buenas prácticas de intervención social con personas sin hogar. Barcelona: Icaria.

Combaluzier, S. y Pedinielli, J. L. (2003). Étude de l'impact des troubles mentaux sur les diffcultés de réinsertion sociale, Annales Médicopsychologiques, 161, 31-37.

Combaluzier, S., Gouvernet, B. y Bernoussi, A. (2009). Impact des troubles de la personnalité dans un échantillon de 212 toxicomanes sans domicile fixe, L'Encéphale, 35, 448-453.

Cosci, F. y Fava, G.A. (2011). New clinical strategies of assessment of comorbidity associated with substance use disorders, Clinical Psychology Review, 31(3), 418-427.

Craig, M., Coldwell, C. M. y Bender, W. S. (2007). The effectiveness of assertive community treatment for homeless populations with severe mental illness: a meta-analysis. American Journal of Psychiatry, 164, 393399.

Csémy, L., Vágnerová, M. M., Marek, J. (2011). Mental disorder in young adult homeless: A review, Psychiatrie, 15(2), 86-92. una mayor psicopatología (INE, 2011; Krausz et al., 2013; Pascual et al., 2008; Notaro et al., 2013). Esta menor prevalencia de trastornos del eje I, puede venir determinada por el alto nivel de deterioro de la persona en la calle (Csémy et al., 2012; Henry et al., 2010; Pascual et al., 2008; Solliday-McRoy et al., 2004), que hace que personas sin hogar con presencia de trastornos del eje I no frecuenten centros de corte psicosocial, permaneciendo en la calle, en numerosas ocasiones sin tratamiento.

Como principal conclusión, la investigación muestra una persona con deterioro cognitivo leve, con trastornos de personalidad del grupo de problemas interpersonales y ambivalentes como los principales candidatos a abandono de procesos de corte psicosocial. Este hecho es coherente con las investigaciones que muestran la comorbilidad entre trastornos como un agente que determina la tasa de abandonos de tratamiento (Cosci y Fava, 2011; Friborg, Martinussen, Kaiser, Overgard y Rosenvinge, 2012; Hayward y Moran, 2008; Salavera et al., 2010), pudiéndose hablar de un factor de complicación del caso y de peor pronóstico, cuando presentan comorbilidad entre ambos ejes.

Ello implica la necesidad de tener en cuenta la comorbilidad entre Ejes en personas sin hogar, tanto en el tratamiento, como en el desarrollo de programas específicos de intervención de tratamiento en la comunidad para favorecer su inserción y disminuir el porcentaje de abandonos (Craig, Coldwell y Bender, 2007; Dixon, Weiden, Torres y Lehman, 1997; Hawkins y Abrams, 2007; Rosenheck, 2000; Wolff, Helminiak, Morse, Calsyn, Klinkenberg y Trusty, 1997).

Por otra parte, el tamaño de la muestra de personas sin hogar, aunque relevante desde el punto de vista clínico, es relativamente pequeño desde una perspectiva estadística. Por ello, se requiere un mayor número de estudios semejantes con el objetivo de poder identificar el perfil específico de trastornos de clínicos y de la personalidad en personas sin hogar.
Dixon, L., Weiden, P., Torres, M. y Lehman, A. (1997). Assertive community treatment and medication compliance in the homeless mentally ill. American Journal of Psychiatry, 154, 1302 - 1304.

Fazel, S., Khosla, V., Doll, H. y Geddes, J. (2008). The prevalence of mental disorders among the homeless in western countries: systematic review and meta-regression analysis, PLoS Medicine, 5(12), e225.

Folsom, D. F., Hawthorne, W., Lindamer, L., Gilmer, T., Bailey, A., Golshan, S., García, P., Unützer, J., Hough, R. y Jeste, D.V. (2005). Prevalence and risk factors for homelessness and utilization of mental health services among 10,340 patients with serious mental illness in a large public mental health system, American Journal of Psychiatry, 162, 370-376.

Friborg, O., Martinussen, M., Kaiser, S., Øvergård, K. T. \& Rosenvinge, J. H. (2013). comorbidity of personality disorders in anxiety disorders: A meta-analysis of 30 years of research, Journal of Affective Disorders, 145 (2), 143-155

Hawkins, R. L. y Abrams, C. (2007). Disappearing acts: The social networks of formely homeless individuals with co-ocurring disorders, Social Science y Medicine, 65, 2031-2042.

Hayward, M. y Moran, P. (2008). Comorbidity of personality disorders and mental illnesses, Psycbiatry, 7 (3), 102-104. 
Henry, J. M., Boyer, L., Belzeaux, R., Baumstarck-Barrau, K. y Samuelian, J. C. (2010). Mental disorders among homeless admitted to a French psychiatric emergency service, Psychiatric Services, 61(3), 264-271.

Herrman, H. (2008). Mental disorders among homeless people in western countries, PLoS Medicine, 5 (12), 1641-1642

Instituto Nacional de Estadística (2011). Encuesta de morbilidad hospitalaria. Madrid: INE.

Instituto Nacional de Estadística (2012). Encuesta a las personas sin hogar. Madrid: INE.

Krausz, R. M., Clarkson, A. F., Strehalu, V., Torchalla, I., Li, K. y Schuetz, C. G. (2013). Mental disorder, service use, and barriers to care among 500 homeless people in 3 different urban settings, Social Psychiatry and Psychiatric Epidemiology, 1-9.

Längle, G., Egerter, B., Albretcht, F., Petrasch, M. y Buchkremer, G. (2005). Prevalence of mental illness among homeless men in the community. Approach to a full census in a southern German university town, Social Psychiatry and Psychiatric Epidemiology, 40, 382-390.

Millon, T. (1997). MCMI-II: Inventario Clínico multiaxial de Millon. Madrid: TEA.

Muñoz, M., Vázquez, C., Bermejo, M., Vázquez, J. J. y Sanz, J. (1996). Trastornos mentales (DSM-III-R) de las personas sin hogar en Madrid: un estudio utilizando la CIDI (Composite International Diagnostic Interview), Archivos de Neurobiología, 59 (4), 269-282.

Muñoz, M., Vázquez, J. J., Panadero, S. y Vázquez, C. (2003). Características de las personas sin hogar en España: 30 años de estudios empíricos, Cuadernos de Psiquiatría Comunitaria, 3 (2), 100-116.

Notaro, S. J., Khan, M., Kim, C., Nasaruddin, M. y Desai, K. (2013). Analysis of the health status of the homeless clients utilizing a free clinic, Journal of Community Health, 38 (1), 172-177.

Pascual, J. C., Malagón, A., Arcega, J. M., Gines, J. M., Navinés, R., Gurrea, A., García-Ribera, C. y Bulbena, A. (2008). Utilization of psychiatric emergency services by homeless persons in Spain, General Hospital Psychiatry, 30 (1), 14-19.

Rosenheck, R. (2000). Cost-Effectiveness of Services for Mentally Ill Homeless People: The Application of Research to Policy and Practice. American Journal of Psychiatry, 157, 1563-1570.
Salavera, C. (2009). Personality disorders in homeless people, International Journal of Psychology and Psychological Therapy, 9 (2), 275-283.

Salavera, C., Puyuelo, M. y Orejudo, S. (2009). Trastornos de personalidad y edad: Estudio con personas sin hogar, Anales de Psicología, 25 (1), 261 265.

Salavera, C., Puyuelo, M., Tricás, J.M. y Lucha, O. (2010). Comorbilidad de trastornos de personalidad: Estudio en personas sin hogar, Universitas Psychologica, 9(2), 457-468.

Samuel, D. B., Connoly, A. J. y Ball, S. A. (2012). The convergent and concurrent validity of trait-based prototype assessment of personality disorder categories in homeless persons, Assessment, 19(3), 287-298.

Speak, S. (2013). Values as a tool for conceptualising homelessness in the global south, Habitat International, 38, 143-149.

Sollliday-McRoy, C., Campbell, T., Melchert, T., Young, T. y Cisler, R. (2004). Neuropsychological functioning of homeless men, The Journal of Nervous and Mental Disease, 192 (7), 471-478.

Sullivan, G., Burnam, A., Koegel, P. y Hollenberg, J. (2000). Quality of life of homeless persons with mental illness: results from the course-ofhomelessness study, Psychiatric Services, 51 (9), 1135-1141.

Twenge, J. M., Baumeister, R. F., DeWall, C. N., Ciarocco, N. J. y Bartels, J. M. (2007). Social exclusion decreases prosocial behavior. Journal of Personality and Social Psychology, 92, 56-66.

Vágnerová, M. M., Csémy, L. y Marek, J. (2012). Personality characteristics of young homeless, Psychiatrie, 16(1), 8-13.

Villeneuve, K., Potvin, S., Lesage, A. y Nicole, L. (2010). Meta-analysis of rates of drop-out from psychosocial treatment among persons with schizophrenia spectrum disorder, Schizophrenia Research, 121 (1-3), 266270.

Wolff, N., Helminiak, T.W., Morse, G. A., Calsyn, R. J., Klinkenberg, W. D. y Trusty, M. L. (1997). Cost-effectiveness evaluation of three approaches to case management for homeless mentally ill clients. American Journal of Psychiatry, 154, 341 - 348.

World Health Organization (1997). Composite International Diagnostic Interview, version 2.1. Geneva: WHO.

(Artículo recibido: 29-3-2012 revisado: 20-02-2013, aceptado: 23-02-2013) 